\title{
Tecnura
}

\section{Entropías de la movilidad urbana en el espacio metropolitano de Guadalajara: Transporte privado y calidad del aire}

\author{
Urban transport entropies in the metropolitan area of Guadalajara: \\ Automobility and air quality
}

\begin{abstract}
Fernando De Quevedo García Najar, ${ }^{1}$ Yefer Asprilla Lara, ${ }^{2}$ Mario Guadalupe González Pérez ${ }^{3}$
\end{abstract}
Fecha de recepción: 19 de julio de 2017

Fecha de aceptación: 18 de noviembre de 2017

Cómo citar: De Quevedo, F., Asprilla, Y. y González, M. (2017). Entropías de la movilidad urbana en el espacio metropolitano de Guadalajara: transporte privado y calidad del aire. Revista Tecnura, 138-149, 21(53), doi: $10.14483 / 22487638.10725$

\section{Resumen}

Contexto: la evidencia empírica demuestra que, en los últimos tres lustros del presente siglo, el parque vehicular se ha incrementado en más de dos millones de unidades en el área metropolitana de Guadalajara. Dentro de este incremento alarmante, muchos de los vehículos no poseen convertidores catalíticos y a pesar de ello circulan cotidianamente por las vías primarias y secundarias de la metrópoli. De ahí el objetivo del presente trabajo consistente en reflexionar sobre la movilidad motorizada que se vive actualmente en la segunda urbe más importante de México. Método: se utiliza un método analítico descriptivo basado en la revisión documental de contenidos temáticos sobre el porcentaje de contaminantes que aporta cada municipio que compone la metrópoli de Guadalajara, con respecto al incremento del parque vehicular, calidad del aire, cantidad de vehículos registrados y cantidad de vehículos verificados. Con base en el enfoque sistémico, se hacen inferencias del escenario actual de la ciudad bajo el argumento de que ésta se encuentra ante la presencia de fuerzas estabilizadoras y desestabilidazoras de su estado homeostático.

Resultados: se obtiene que la política gubernamental en materia de reducción de contaminantes ha tenido poco impacto en la concientización de la población.Por lo tanto, resulta necesario realizar un repensamiento de las acciones gubernamentales y ciudadanas para reducir la probabilidad de precontingencias y contingencias ambientales y promover la cultura de la movilidad incluyente, sustentable y segura. Asimismo, los datos sugieren que existe una ausencia sistémica en la planificación de la ciudad y del territorio.

Conclusiones: la ausencia sistémica en la planificación de la ciudad y el territorio ha originado condiciones hostiles manifiestas en el espacio físico. En este espacio, los vehículos privados, y en general los medios motorizados, se han convertido en estas fuerzas que alteran la homeostasis de la ciudad provocando entropía, lo que conlleva al uso de medios de transporte sostenibles y a trabajar colectivamente para la sustitución sistemática del automóvil.

1 Ingeniero civil, magister in Science in Civil Enginereeng, estudiante de doctorado en Movilidad, Urbana, Transporte y Territorio. Profesor del Instituto Tecnológico de Monterrey. Guadalajara, México. Contacto: fdoquevedo@itesm.mx

2 Ingeniero civil, especialista en Infraestructura Vial y Transporte, magister en Ingeniería Civil, estudiante doctorado en Movilidad, Urbana, Transporte y Territorio. Profesor asociado de la Universidad Distrital Francisco José de Caldas. Bogotá D.C., Colombia.

Contacto: yasprillal@udistrital.edu.co

3 Ingeniero civil, magister en Ingeniería Civil, doctor en Ciudad, Territorio y Sustentabilidad. Profesor Titular de la Universidad de Guadalajara. México. Contacto: mario.Gonzalez@academico.udg.mx 
Palabras clave: automobilidad, calidad del aire, entropía, movilidad, sostenibilidad.

\section{Abstract}

Context: Empirical evidence shows that vehicle ownership has increased exponentially in the last fifteen years in the Metropolitan Area of Guadalajara, amounting more than two million vehicles. Moreover, a high percentage of these vehicles is very old and lack air-pollution control systems such as catalytic converters. The goal of this paper is to meditate about motorized transport in the second largest city in Mexico.

Method: A descriptive analytical method was used to review data and information from governmental and non-governmental sources. Air contaminants are related to car ownership increment, county multi-modal transport distribution, registered vehicles, and the amount of vehicles that have been verified and certified by the government air quality program. From a systems theory point of view, current scenarios are analyzed and inferences are made. As a result, there are balancing and unbalancing forces that affect the homeostasis of this metropolitan area.
Results: Air-quality regulations have failed to echo among their citizens that neglect to tune up and verify emissions from their vehicles. Therefore, government officials and the society itself must reconsider their efforts and renew air quality policies based on low emissions, safe, reliable, and sustainable transportation to avoid air quality contingencies. In addition, data clearly suggests that land and urbanistic planning has not followed a systemic approach.

Conclusions: Guadalajara metropolitan area clearly shows a lack of a systemic methodology in planning its urbanistic and land development. Hence, a perverse synergy of hostile conditions have emerged within its territory and internal combustion vehicles are the most important destabilizing force in the homeostasis of the city increasing entropy. The presence of this entropy is forcing the inhabitants in Guadalajara to migrate to a multi-modal sustainable transportation system, and start a systematic car substitution program that ensures zero or very low vehicle emissions.

Keywords: air quality, automobilty, entropies, sustainability, transport.

\section{INTRODUCCIÓN}

En materia vial, diferentes ciudades de México han emprendido en las últimas tres décadas acciones concentradas en la construcción de infraestructura para la movilidad urbana y periurbana tales como: ampliación de avenidas y calles, ampliación de vialidades, pasos a desnivel y hasta la construcción de autopistas urbanas elevadas denominadas segundos pisos. Esta política de infraestructura ha estado enfocada principalmente en la promoción y mejoramiento de la movilidad motorizada de los vehículos privados, sobre todo, porque "en gran parte de estos centros urbanos, particularmente en aquellos que pertenecen a los países en vías de desarrollo, el número de habitantes de los denominados tugurios asciende a más del $50 \%$ de la población" (Valbuena, García y Granados, 2017, p. 80), lo que ha llevado a un incremento desproporcional del parque vehicular respecto al aumento poblacional, pues mientras la población creció el $77,11 \%$ entre 1980 y 2015, el crecimiento vehicular aumentó hasta un 536\%, tal y como se presenta en la figura 1.

En función de lo anterior, resulta plausible suponer que existe una ausencia sistémica en la planificación de la ciudad y el territorio que ha generado un caldo de combinaciones de elementos de difícil determinación morfológica. Algunas de estas combinaciones generan condiciones hostiles manifiestas en el espacio físico, lo que se vuelve aún más complejo, no solo por la insuficiencia o deficiencia de infraestructura física, sino por la conformación de fuerzas que trastocan constantemente la 


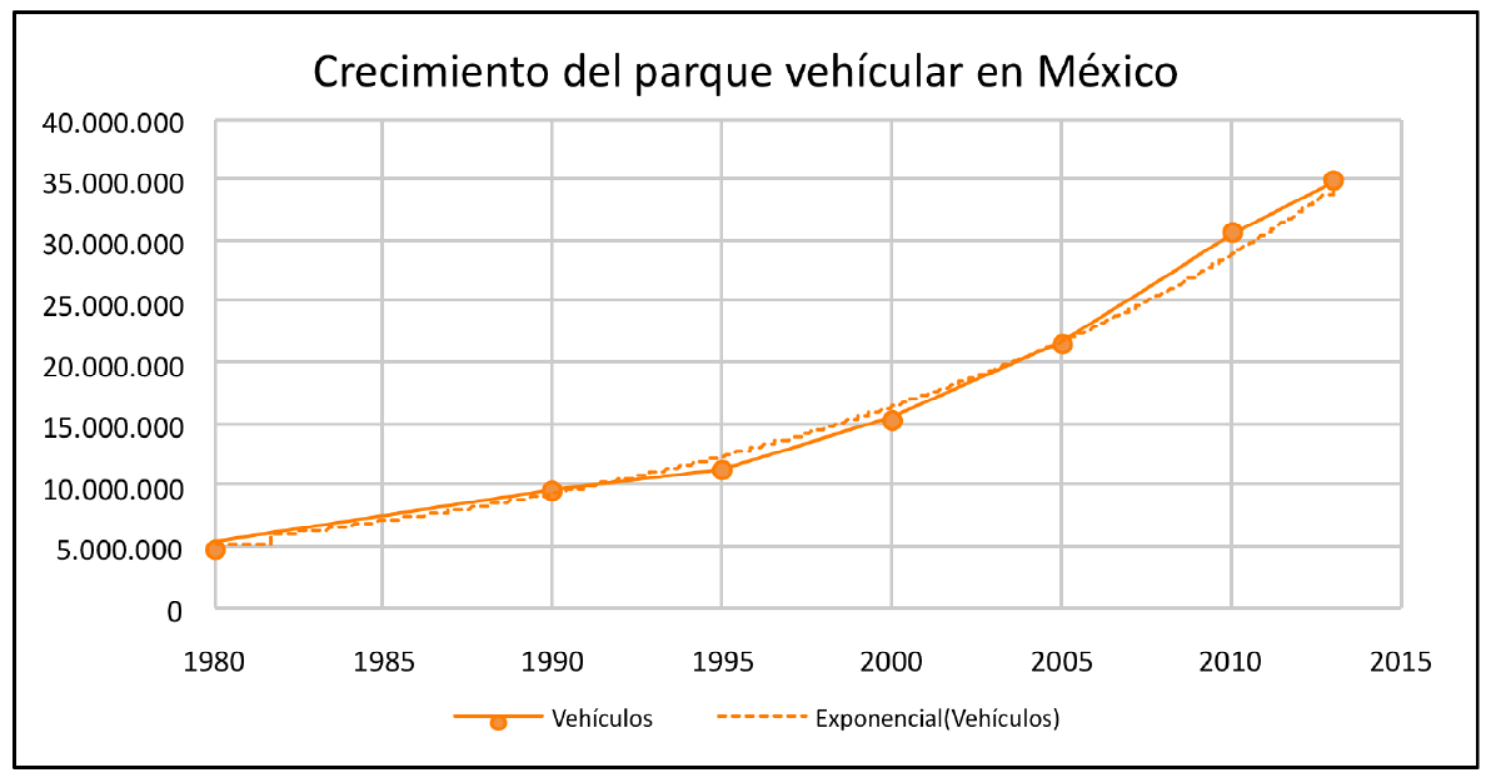

Figura 1. Crecimiento del registro de vehículos en México

Fuente: elaboración propia con datos de (Cervera y Rangel, 2015)

homeostasia de la ciudad generando entropía, es decir, desorden ambiental, económico y social en el sistema (González y Asprilla, 2016; González, 2017). Sin embargo, en contraposición al proceso de desestabilización causado por las fuerzas entrópicas, existen también, un conjunto de fuerzas que pretenden el efecto contrario y son conocidas comúnmente como fuerzas neguentrópicas o intervenciones in situ de origen antropogénico tendientes a disminuir el malestar urbano. En suma, "la insuficiencia y deficiencia de conectividad, accesibilidad y medios adecuados de transporte han representado altos costos para la movilidad urbana" (González, 2016, p. 25).

En este contexto, ha sido una interrogante frecuente el indagar la ocurrencia del crecimiento vehicular, sobre todo porque la complejidad de la respuesta ha conducido a una multifactorialidad combinada, tanto por la disminución de las tasas de interés de las instituciones crediticias nacionales para la compra de vehículos nuevos y seminuevos, como por el componente aspiracional de los individuos de clase baja y media relacionado con la posesión de un vehículo privado (estatus), una mayor seguridad, o por el deficiente e insuficiente transporte público, el cual resulta menos práctico que la utilización del vehículo privado. No obstante, la causal principal recae en el ejercicio de la política pública federal asumida desde el sexenio del expresidente Vicente Fox Quezada (20002006), debido a que permitió la importación de automóviles y camionetas de pasajeros provenientes del extranjero, principalmente de Estados Unidos y Canadá.

Esta política pública le facilitó a la población de bajo poder adquisitivo adquirir un vehículo particular, generalmente camionetas pequeñas tipo pick up; incluso, tan solo el último año de ese sexenio, se estima que la introducción de vehículos usados alcanzó la cifra de 1121731 unidades (Sipse, 2014). En este sentido, estas acciones coadyuvaron con la consolidación del imaginario social que le otorga al automóvil la cualidad de ser el modo de transporte más flexible, rápido y 
confortable; efectivamente, pudieron acceder a ellos muchos mexicanos de clase media y media baja, sin embargo, el costo para la movilidad urbana fue demasiado alto. Toda vez que mientras en 1980 se tenía un vehículo por cada doce habitantes, en 2013 la cifra pasó a un vehículo por cada tres habitantes (Cervera y Rangel, 2015).

En el área metropolitana de Guadalajara (AMG), los esfuerzos por la promoción del transporte público masivo se han limitado únicamente a la operación de una sola línea del autobús articulado de tránsito rápido (BRT), dos líneas de tren eléctrico urbano en funcionamiento, y una más en proceso de construcción (línea tres), con intenciones de comenzar operaciones a finales del 2018; no obstante, estos sistemas no se encuentran integrados al servicio de transporte colectivo prestado por los buses urbanos (camiones), lo que dificulta aún más la movilidad para los usuarios del transporte público, caso contrario sucede en otras ciudades de la región, como por ejemplo en Bogotá D.C., donde la implementación del Sistema Integrado de Transporte Público conocido como SITP, ha permitido que con un único medio de pago (tarjeta Tu llave) se pueda realizar el transbordo entre los diferentes servicios de buses de la ciudad (Asprilla y Rey, 2012).

La movilidad de los habitantes del AMG y sus alrededores se ha basado principalmente en vehículos motorizados de combustión interna de baja capacidad, de ahí que el resultado de esta modalidad basada en el automóvil y el autotransporte público de bajo volumen (autobuses urbanos) hayan generado grandes externalidades como: accidentalidad, mortalidad, morbilidad, congestionamiento vial, pérdida de horas efectivas, ruido y emisiones atmosféricas de contaminantes que afectan las características del aire y, en consecuencia, la salud de las personas. Además, se estima que la movilidad en vehículo privado representa el $40 \%$ de los viajes diarios en comparación con el área metropolitana del Valle de México, donde la movilidad en automóvil solo representaba el 29\% del total diario (ONU-Hábitat, 2015).
A lo anterior también hay que adherir la existencia de una gran proporción de vehículos que superan los diez años de antigüedad, lo que implica que estas unidades no cuenten con convertidores catalíticos en sus sistemas de escape para el control de emisiones; esto conduce a que la eficiencia de sus motores tienda a ser muy baja respecto al consumo de combustibles fósiles. Según Cervera y Rangel (2015), la antigüedad promedio de los vehículos en el país oscilaba en los dieciocho años de antigüedad, cantidad que se aproxima a lo reportado por Melgar y Asociados sobre los 16,58 y 16,70 años en promedio (Portal Automotriz, 2012), o al Diario Oficial de la Federación (2009), quien le otorgaba al parque vehicular una antigüedad de 16,3 años.

Es importante distinguir que este estudio clasifica al parque vehicular en tres categorías: los vehículos que entran en forma ilegal con una antigüedad promedio de 19,53 años, los vehículos importados legalizados con una antigüedad de 18.01 años y los vehículos comercializados en México con un promedio de edad de 12,12 años; en este sentido, el trabajo relaciona los estudios sobre movilidad y sistemas de transporte urbano motorizado. A nivel general se preocupa por el uso excesivo del vehículo privado que está incidiendo fuertemente en la emisión de partículas contaminantes, por ello, el objetivo central plantea "reflexionar sobre el escenario de la movilidad motorizada que está experimentado actualmente el Área Metropolitana de Guadalajara"; de aquí, se hace uso metodológicamente del método analítico descriptivo con base en la revisión y análisis de contenidos temáticos de fuentes estadísticas primarias e información de fuentes secundarias a nivel nacional, estatal y local.

Covarrubias (2014), en "La explosión de la Industria Automotriz en México", afirma que el tiempo máximo de operación de un vehículo de manera eficiente es de diez años en promedio, y esto en condiciones de mantenimiento preventivo y correctivo óptimo, ya que después de ese periodo los costos de mantenimiento en unidad y su 
nivel de emisiones aumentan un $20 \%$ más en promedio por cada año adicional de operación.

En México, se han efectuado inventarios de emisiones de contaminantes tales como: cantidades de óxidos de nitrógeno y azufre $\left(\mathrm{NO}_{x}\right.$ y $\left.\mathrm{SO}_{2}\right)$, combinaciones orgánicas de tipo volátil (COV), presencia en el aire de partículas suspendidas (PM), monóxido de Carbono (CO) o amoniaco $\left(\mathrm{NH}_{3}\right)$; en efecto, la mayoría de estos gases son producto de la quema de los combustibles utilizados en la movilidad motorizada, lo que ha conducido a que los vehículos se conviertan en los principales responsables de más del $85 \%$ de todos los contaminantes, pero especialmente de $\mathrm{CO}, \mathrm{NOx}$ y los COV. El otro $15 \%$ restante proviene de fuentes fijas, naturales y de área (SEMADET, 2013).

\section{METODOLOGÍA}

El ejercicio metodológico hace uso del análisis descriptivo a través de la revisión documental de los indicadores de los organismos de monitoreo ambiental, investigaciones científicas y datos vehiculares de las principales empresas automotrices de México; para esto, se ha identificado al parque vehicular en tres categorías: vehículos que entran ilegalmente al país con una antigüedad promedio de 19,53 años, vehículos importados legalizados con una antigüedad de 18,01 años y vehículos comercializados por la industria automotriz con un promedio de edad de 12,12 años. El análisis ha considerado también:

- El porcentaje de contaminantes que aporta cada municipio que compone el AMG, respecto al incremento del parque vehicular.

- Calidad del aire.

- Cantidad de vehículos registrados.

- Cantidad de vehículos verificados.

Por último, se reflexiona desde un enfoque sistémico el escenario actual de la ciudad, toda vez que la ciudad concebida como un sistema se encuentra sujeta a la presión de fuerzas de orden o de composición bariónica y fuerzas de desorden o de composición fotónica (Prigogine, 1983), en resumen: "el resultado de este conflicto entre fuerzas fotónicas y bariónicas se manifiesta en un fuerte deterioro ambiental" (González y Jalomo, 2017); de aquí, el énfasis sobre la necesidad de fomentar la práctica de la movilidad urbana sostenible y reducir paulatinamente el consumo del vehículo privado.

En el AMG, el Colectivo Ecologista Jalisco AC y Latin American Capital (2013) sostienen que los municipios más desarrollados económicamente, como lo son Guadalajara y Zapopan, serían los principales contribuyentes de contaminantes, conforme se expone en la figura 2; además, esto coincide con el grado de desarrollo del parque vehicular de estos dos municipios que concentraban 1317181 vehículos de un total de 1729961 vehículos existentes en ese año en toda la metrópoli.

Los autobuses, automóviles, camionetas y otros medios de transporte motorizado representan el $76,14 \%$ de todo el parque vehicular metropolitano; las fuentes de emisión de contaminantes como las del transporte, aunado a las fuentes fijas originadas por las instalaciones industriales y comerciales han obligado a la SEMADET a instrumentar un monitoreo del aire considerando los contaminantes anteriormente mencionados, denominado Sistema de Monitoreo Atmosférico de Jalisco (SIMAJ). Este organismo cuenta con diez estaciones fijas, pero solo cubrían los municipios que componen la zona metropolitana (Guadalajara, Zapopan, Tonalá, Tlaquepaque, y Tlajomulco de Zúñiga), excluyendo las cabeceras municipales de El Salto, Juanacatlán, Ixtlahuacán de los Membrillos y Zapotlanejo (Colectivo Ecologista Jalisco AC y Latin American Capital, 2013).

De acuerdo con la SEMADET (2016), el SIMAJ se basa en la concentración de contaminantes, donde ha generado un índice metropolitano de calidad de aire (IMECA) que puede fluctuar entre cero y más de 200 puntos, dependiendo del valor indicado se establece un índice de calidad del aire. Así, un IMECA de 0 a 50 puntos representa una buena calidad de aire; de 51 a 100, se considera 


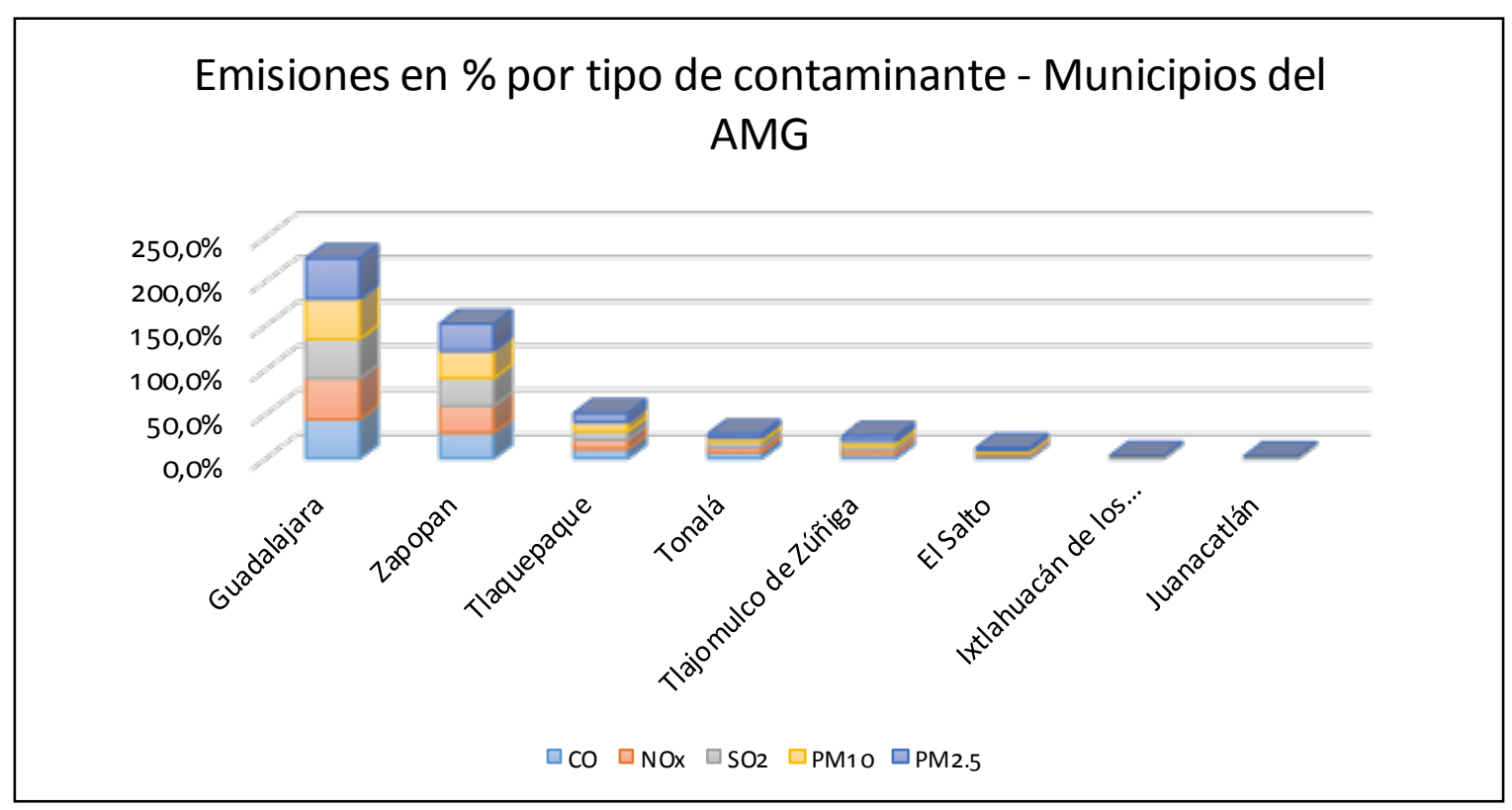

Figura 2. Distribución porcentual de contaminantes criterio por Municipio

Fuente: elaboración propia con datos de (Colectivo Ecologista Jalisco AC., y Latino American Capital, 2013)

regular; de 101 a 150, mala calidad y valores mayores de 151 representan una calidad muy mala. EI IMECA genera una serie de indicaciones para la población en general, enfocada en actividades al aire libre; sin embargo, el AMG ha sufrido contingencias ambientales esporádicas con episodios de calidad de aire mala y muy mala en los últimos años, de acuerdo con lo expuesto en la tabla 1.

La información de la tabla 1 constata que la calidad del aire en el AMG está disminuyendo importantemente, dado que se están presentando mayores precontingencias y contingencias denominadas Fase 1; además, la negligencia de la población para afinar y verificar la emisión de contaminantes de los vehículos se ha acentuado en los últimos cinco años, donde la verificación no supera el $25 \%$ de los vehículos registrados a nivel estatal de acuerdo con lo presentado en la figura 3 .

Tabla 1. Calidad del aire en días para los años 2010 al 2014

\begin{tabular}{|c|c|c|c|c|c|c|}
\hline \multicolumn{7}{|c|}{ Días del año con calidad de aire } \\
\hline IMECA & Bueno & Regular & Malo & Muy malo & $\begin{array}{c}\text { Extremadamente } \\
\text { Mala }\end{array}$ & $\begin{array}{c}\text { Contaminante Criterio/ } \\
\text { Estación }\end{array}$ \\
\hline 2010 & No se reporta & No se reporta & 133 & 18 & 1 & Ozono, estación Tlaquepaque \\
\hline 2011 & No se reporta & No se reporta & 237 & 32 & 3 & Ozono, Estación Las Pintas \\
\hline 2012 & No se reporta & No se reporta & 128 & 9 & 2 & PM10, estación Las Pintas \\
\hline 2013 & 27 & 220 & 107 & 12 & 0 & Ozono, Estación Atemajac \\
\hline 2014 & 239 & 0 & 123 & 3 & 0 & PM10, Estación Santa Fe \\
\hline
\end{tabular}

Fuente: elaboración propia con datos de (SEMADET, 2016) 


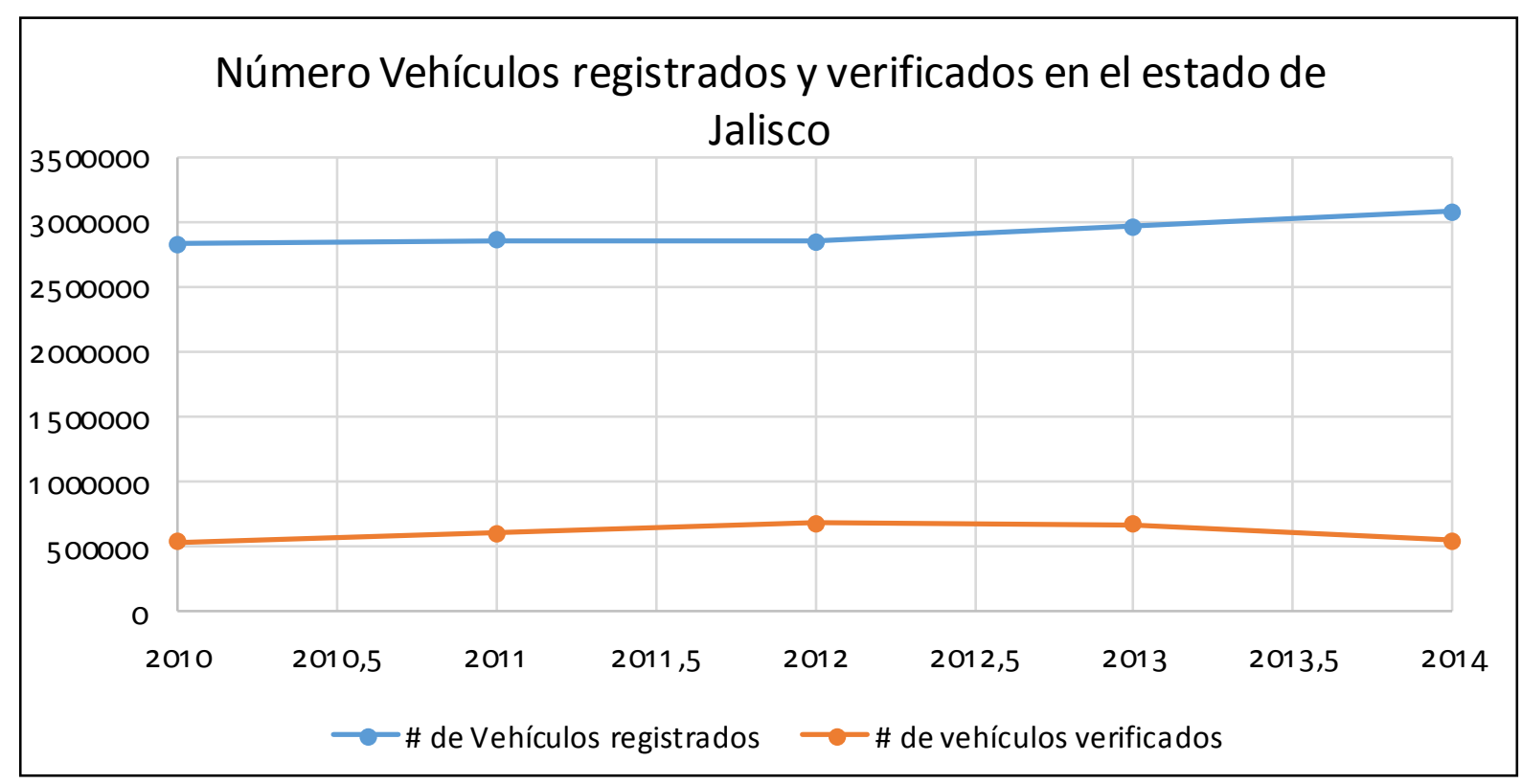

Figura 3. Número de vehículos registrados y número de vehículos verificados

Fuente: elaboración propia con datos de (SEMADET, 2013 y 2016)

Ciertamente, no es de sorprender que las contingencias y precontingencias ambientales sean más frecuentes cada año, con repercusiones importantes en la salud de los ciudadanos, ejemplo de ello es el estudio del Instituto de Astronomía y Meteorología (IAM), a través de su director Hermes Ulises Ramírez Sánchez, quien observó que en el primer quinquenio de este siglo, los padecimientos respiratorios de infantes menores a los cinco años de edad, pudieran estar asociados con las condiciones de calidad del aire reportadas por el SIMAJ (Ramírez, 2006); adicionalmente, se han dado señalamientos por parte de la jefatura de infectología del Hospital Civil de Guadalajara referentes a las enfermedades obstructivas crónicas, las cuales se incrementan cada día, y esto incluye también a niños, en este caso, la contaminación ambiental es el único factor para ello (El Informador, 2016).

Destacan también, las investigaciones realizadas por la Universidad de Guadalajara, mediante el trabajo de Garibay, Herrera y Curiel, quienes sostienen que desde principios de este siglo la calidad del aire se distancia cada vez más de lo aceptable, incrementándose el número de personas expuestas a concentraciones peligrosas, a grado tal de llegar hasta el doble de muertes por enfermedades respiratorias cada dieciséis años (Carrillo, 2009). En este sentido, de acuerdo con el Instituto Nacional de Ecología y Cambio Climático (2014), la población del AMG está padeciendo afectaciones a la salud identificada por un deterioro en su calidad de vida, vinculada como se ha mencionado con la contaminación de fuentes móviles y estacionarias como las ladrilleras en la periferia metropolitana, donde el hollín se convierte en el principal contaminante, pues no solo deteriora la salud, sino que incrementa el efecto de calentamiento global.

\section{RESULTADOS}

En la actualidad, la industria automotriz ofrece alternativas tecnológicas para reducir las emisiones atmosféricas de todo tipo de vehículos comenzando por motores de bajo cilindraje, uso de turbo cargadores, motores híbridos y vehículos completamente eléctricos. De hecho, "el vehículo 
eléctrico actualmente es parte de una política de transporte sostenible y eficiente que busca disminuir el consumo de combustibles fósiles" (Mendoza, Quintero y Alarcón, 2015, p. 91). Incluso, ciudades como Melbourne en Australia, se ha propuesto la meta de reducir las emisiones de carbono a cero para el 2020 (City of Melbourne, 2016) y Paris para el año 2050 (Wiseman, 2015). Esto basado en un transporte público eléctrico, movilidad no motorizada y, por supuesto, tecnologías automotrices limpias.

Las compañías manufactureras de automóviles están enfocando sus esfuerzos de investigación en la producción de vehículos más eficientes y amigables con el medio ambiente natural, a través de automóviles eléctricos que generan cero emisiones durante su operación; adicionalmente, se ha aumentado la diversidad y la producción de vehículos híbridos, es decir, combinan un pequeño y eficiente motor de combustión interna y uno eléctrico que lleva rendimientos combinados de más de $40 \mathrm{~km}$ por litro de combustible, como es el caso del Toyota Prius (Nexu, 2016).

En el caso de México se tienen 34 marcas automotrices que comercializan vehículos automotores, de los cuales se tienen unidades eléctricas, híbridas y modelos con motorizaciones más bajas y menor generación de emisiones de gases a la atmósfera; sin embargo, en muchos casos, las empresas del ramo automotriz no reportan la cantidad de gases emitidos por sus vehículos, pues solo once de las 34 comparten la información referente a la producción de gramos $\mathrm{CO}_{2}$ por km. Entre las marcas que sí reportan esta información, se encuentran los fabricantes alemanes Mercedes Benz, BMW, Volkswagen, Porsche y Smart, la francesa Peugeot, las japonesas Mazda y Subaru y, finalmente, la coreana Kia, tal y como se señalan en la tabla 2 .

Tabla 2. Relación de fabricantes automotrices comercializados en México

\begin{tabular}{|c|c|c|c|c|c|}
\hline Marca & Modelo & Motor & $\begin{array}{c}\text { Precio en } \\
\text { pesos mexicanos }\end{array}$ & $\begin{array}{c}\text { Emisiones de } \mathrm{CO}^{2} \\
\text { ciclo mixto } \\
(\mathrm{g} / \mathrm{km})\end{array}$ & $\begin{array}{l}\text { Emisiones según } \\
\text { Eco Vehículos }\end{array}$ \\
\hline Nissan & Leaf & Eléctrico & 601200 & No emite emisiones & \\
\hline Renault & Twizy & Eléctrico & 290000 & No emite emisiones & \\
\hline Audi & A1 e-tron & Eléctrico & No se reporta & No emite emisiones & \\
\hline $\begin{array}{l}\text { Mercedes } \\
\text { Benz }\end{array}$ & Clase A & combustión interna & desde 444900 & $129-125$ & 174 modelo 2013 \\
\hline BMW & i3 & Eléctrico & 699900 & No emite emisiones & \\
\hline BMW & i8 & Eléctrico & 2501900 & No emite emisiones & \\
\hline $\mathrm{BMW}$ & $320 \mathrm{i}$ & combustión interna & desde 514900 & 129.1 & 189 \\
\hline Volkswagen & UP & combustión interna & desde 146900 & 129 & No se enlista modelo \\
\hline Chevrolet & Volt & Eléctrico & 657800 & No emite emisiones & 122 híbrido \\
\hline Dodge & Attitude & combustión interna & desde 168900 & Sin reporte & 186 modelo 2014 \\
\hline Chrysler & 200 & combustión interna & desde 345000 & Sin reporte & 244 modelo 2014 \\
\hline Fiat & Uno & combustión interna & desde 178900 & Sin reporte & 228 modelo 2014 \\
\hline Jeep & Patriot & combustión interna & desde 302400 & Sin reporte & $\begin{array}{l}208 \text { modelo } 2015 \\
\text { TM }\end{array}$ \\
\hline Ram & 700 & combustión interna & desde 177900 & Sin reporte & $\begin{array}{l}234 \text { modelo } 2014 \\
\text { (FIAT Strada) }\end{array}$ \\
\hline Mitsubishi & ASX & $\begin{array}{l}\text { combustiona } \\
\text { interna }\end{array}$ & desde 322900 & Sin reporte & 206 \\
\hline Honda & Civic & combustión interna & desde 292900 & Sin reporte & 181 \\
\hline Accura & ILX & combustión interna & desde 469900 & Sin reporte & 195 \\
\hline
\end{tabular}




\begin{tabular}{|c|c|c|c|c|c|}
\hline Marca & Modelo & Motor & $\begin{array}{c}\text { Precio en } \\
\text { pesos mexicanos }\end{array}$ & $\begin{array}{c}\text { Emisiones de } \mathrm{CO}^{2} \\
\text { ciclo mixto } \\
(\mathrm{g} / \mathrm{km})\end{array}$ & $\begin{array}{l}\text { Emisiones según } \\
\text { Eco Vehículos }\end{array}$ \\
\hline Toyota & Prius & Hibrido & desde 339700 & Sin reporte & 147 modelo 2014 \\
\hline Mazda & CX-3 & combustión interna & desde 334900 & 134.2 & 164 \\
\hline Subaru & Forester 2.0i XT & combustión interna & desde 595.900 & 190 & 203 \\
\hline INFINITI & Q50 & Hibrido & 720200 & Sin reporte & No se enlista modelo \\
\hline INFINITI & QX60 & Hibrido & 1039900 & Sin reporte & No se enlista modelo \\
\hline $\mathrm{KIA}$ & Soul LX & combustión interna & desde 238900 & 160.31 & No se enlista modelo \\
\hline HYUNDAI & Grand i10 & combustión interna & desde 143150 & Sin reporte & 166 \\
\hline BUICK & Encore & combustión interna & desde 397200 & Sin reporte & 205 \\
\hline GMC & Terrain & combustión interna & desde 504200 & Sin reporte & 280 \\
\hline Cadillac & ATS Sedan & combustión interna & desde 707300 & Sin reporte & 216 \\
\hline Peugeot & 301 Sedan Diésel & $\begin{array}{l}\text { combustión interna } \\
\text { diésel }\end{array}$ & desde 236900 & 119 & No se enlista modelo \\
\hline Volvo & V40 T3 & combustión interna & desde 379900 & 127 & $\begin{array}{l}185 \text { modelo } 2014 \\
\text { TM }\end{array}$ \\
\hline Jaguar & XF 2.0i4 Turbo & combustión interna & desde 59900 (dólares) & 104 & 366 \\
\hline Landrover & Discover Sport & combustión interna & desde 44900 (dólares) & Sin reporte & No se enlista modelo \\
\hline Porsche & Macan S Diesel & combustión interna & desde 947600 & $164 \mathrm{~g}-159 \mathrm{~g}$ & 209 \\
\hline Porsche & $\begin{array}{l}\text { Panamera S-E } \\
\text { Hybrid }\end{array}$ & Hibrido & desde 1714500 & 71 & 209 \\
\hline Alfa Romeo & Mito Cuadrifolglio & combustión interna & desde 356900 & Sin reporte & $\begin{array}{l}192 \text { modelo } 2014 \\
\text { TM }\end{array}$ \\
\hline Seat & Ibiza FR & combustión interna & desde 185700 & 113 & $\begin{array}{l}149 \text { modelo } 2015 \\
\text { TM, Turbo }\end{array}$ \\
\hline Smart & ForTwo & combustión interna & desde 198900 & 93 & $\begin{array}{l}154 \text { modelo } 2014 \\
\text { TA, Turbo }\end{array}$ \\
\hline Suzuki & Swift & combustión interna & desde 174000 & Sin reporte & $\begin{array}{l}171 \text { modelo } 2014 \\
\text { TA, TM }\end{array}$ \\
\hline Lincoln & MKZ & combustión interna & desde 573500 & Sin reporte & 221 \\
\hline
\end{tabular}

Fuente: elaboración propia con datos de los 34 fabricantes y del Portal de Indicadores de Eficiencia Energética y Emisiones Vehiculares de Eco Vehículos, 2016

Una característica importante que se debe destacar es que la oferta de vehículos eléctricos en México ya asciende a seis modelos de vehículos, que incluye dos modelos del fabricante alemán BMW, uno más de la también empresa alemana Audi, un modelo de la japonesa Nissan, otro modelo de la francesa Renault y la norteamericana Chevrolet. El precio de estos vehículos oscila entre los 290000 pesos mexicanos para el biplaza Renault Twizy y los 2501900 pesos mexicanos para el $B M W$ i8, considerando solo cinco modelos, ya que Audi no reporta su precio, es decir, el rango oscila entre los 15 425,5 a los 133 079,8 dólares estadounidenses, con un valor de un dólar equivalente a 18,8 pesos mexicanos. En el caso de los vehículos híbridos reportados por las comercializadoras se cuentan con tres modelos de los fabricantes japoneses: Toyota e Infinity con precios que oscilan entre los \$ 339700 pesos mexicanos para el Toyota Prius (18 069 dólares estadounidenses), y de \$ 1039900 para el lujoso Infinity QX60 (55 314 dólares estadounidenses). Estos precios de venta están lejos de las posibilidades de compra de la mayoría de la población mexicana.

La consulta del Portal de indicadores de eficiencia energética y emisiones vehiculares arroja datos que permiten determinar los vehículos más verdes ambientalmente hablando, o los que menos 
emisiones arrojan a la atmosfera, así como los que producen mayores gases, considerando dos criterios de calificación de las emisiones: "vehículos con calificación de contaminantes al aire y vehículos con calificación de gases de efecto invernadero" (Eco vehículos, 2016). Los resultados de los modelos de autos con las más altas calificaciones en ambos criterios solo califican a los vehículos híbridos y eléctricos; sin embargo, se tienen tres modelos con motores de combustión interna que tienen la característica común de contar con motores de bajo cilindraje (menor de 1.4 litros por $\mathrm{km})$, y en algunos casos la inclusión de un turbo cargador que emiten menores cantidades: Smart For two, Seat Ibiza y Chevrolet Spark; conforme se indica en la tabla 3.

Tabla 3. Vehículos comerciales con menores emisiones atmosféricas en México

\begin{tabular}{lllcccc}
\hline \multicolumn{1}{c}{ Marca } & Submarca & \multicolumn{1}{c}{ Versión } & Modelo & $\begin{array}{c}\text { Rendimiento } \\
\text { Combinado } \\
\text { (km/l) }\end{array}$ & $\begin{array}{c}\mathbf{C O}_{\mathbf{2}} \\
(\mathbf{g} / \mathbf{k m})\end{array}$ & $\begin{array}{c}\text { NOx } \\
(\mathbf{g} / \mathbf{1 0 0 0} \mathbf{k m})\end{array}$ \\
\hline HONDA & CIVIC & Híbrido 4 pts 1.5 litros, 4cil, 90 hp & 2014 & 26.15 & 119 & 7 \\
\hline FORD & FUSION & Hibrido 4pts 2.0 litros, 4cil 188 hp & 2015 & 28.93 & 107 & 5 \\
\hline CHEVROLET & SPARK & Ev eléctrico 5pts 139 hp & 2016 & 72.21 & 43 & 0 \\
\hline CHEVROLET & VOLT & Sedan hibrido 4pts 1.5 litros, 4cil 100 hp & 2016 & 25.4 & 122 & 2 \\
\hline
\end{tabular}

Fuente: elaboración propia con datos del manual del usuario del portal de Indicadores de Eficiencia Energética y Emisiones Vehiculares de Eco vehículos, 2016

La tecnología automotriz a nivel mundial ha trabajado en la mitigación de gases contaminantes de efecto directo en la salud de los habitantes urbanos; continuamente, las nuevas opciones son más eficientes, más ligeras y en muchas ocasiones de menor cilindraje que se compensa con turbocompresores para brindar una potencia suficiente para una movilidad segura y rápida. Evidentemente, la inclusión de automóviles híbridos y eléctricos en México sigue siendo muy lenta, debido a sus altos precios de mercado.

\section{CONCLUSIONES}

El AMG enfrenta un reto sin precedentes: el de evitar que la salud de sus habitantes se continué deteriorando por la mala calidad del aire; en este sentido, las autoridades y ciudadanía deben trabajar en forma conjunta para disminuir las emisiones de gases provenientes de actividades económicas industrializadas y las generadas por la movilidad motorizada, que en conjunto representan fuerzas desestabilizadoras del metabolismo urbano. Una solución integral solo será posible con una socialización real y efectiva de las directrices estipuladas en la Ley y Reglamento de movilidad estatales, los cuales promueven un transporte público multimodal, incluyente, eficiente, seguro, amigable con el medio ambiente natural, es decir, que bajo la articulación de los sistemas de transporte masivo sustentables (tren ligero, BRT y autobuses de bajas emisiones), la movilidad cotidiana de distancias medias y cortas estaría complementada con una movilidad ciclista y peatonal de gran cobertura.

En la actualidad existen ejemplos exitosos que promueven la migración hacia una movilidad no motorizada, y quizá el más notorio lo constituye Nueva Delhi en la India, donde la apertura del metro logró en pocos años reducir el tráfico automotriz en casi el $40 \%$, debido a una movilidad más expedita y económica para el usuario, particularmente el que se encuentra circunscrito en la clase media (Siemiatycki, 2006). 
Efectivamente, el AMG es un área dinámica y de alta participación en la economía del país, es un núcleo importante de la cultura, el deporte, el comercio y la identidad nacional que equívocamente ha favorecido el paradigma del automóvil; sin embargo, se está en tiempo de emprender acciones gubernamentales, pero sobre todo ciudadanas para reducir la probabilidad de precontingencias y contingencias ambientales que afecten las expectativas de vida de los ciudadanos y que ocasionan un alto costo social y económico. Por tanto, el compromiso general recae en la promoción de la cultura de la movilidad incluyente, sustentable y segura. De igual forma, fomentar el respeto y la convivencia con los otros modos de transporte, pero, sobre todo, trabajar hacia el reemplazo sistemático del automóvil, debido a que este paradigma ya no es la alternativa más eficiente, sino al contrario, se ha convertido en un problema metropolitano de enorme complejidad. El reto, es la consolidación del paradigma de la movilidad sostenible, toda vez que el uso intensivo del automóvil favorece la generación de entropía en la ciudad, y en consecuencia caos vial, accidentalidad, ruido, contaminación y exclusión de los otros modos de la movilidad.

\section{REFERENCIAS}

Asprilla, Y. y Rey E. (2012). La implementación del Sistema Integrado de Transporte Público (SITP) de Bogotá y sus retos en el futuro. Tecnogestión 9(1), 26-40. Recuperado de http://revistas.udistrital.edu. co/ojs/index.php/tecges /article/view/5649/7167

Carrillo, J. (2009). Rebasa la contaminación del aire en la ZMG los estándares de la OMS. Universidad de Guadalajara. Recuperado de http://www.udg.mx/ es/noticia/rebasa-la-contaminacion-del-aire-en-lazmg-los-estandares-de-la-oms

Cervera, M. y Rangel, J. (2015). Distribución de la población por tamaño de localidad y su relación con el medio ambiente. Seminario Taller "Información para la Toma de Decisiones". México: El
Colegio de México. Recuperado de www.inegi.org. $m x>$ doc s $p$-WalterRangel

City of Melbourne. (2016). Zero Net Emissions Strategy. Recuperado de www.melbourne.vic.gov.au/SiteCoIlectionDocuments/zero-net-emissions-2002.pdf

Colectivo ecologista Jalisco A.C. y Latin American Capital. (2013). Costos económicos y ambientales en el Área Metropolitana de Guadalajara. Recuperado de ciudadpixel.mx documentos

Covarrubias, A. (2014). La explosión de la Industria Automotriz en México. Friedrich Abert Stiftung, 1. Recuperado de http://www.fesmex.org/common/ Documentos/Libros/Paper_AP_Explosion_dela_ Ind_Automortriz_AlexCovarrubias_Mar2014.pdf

Diario Oficial de la Federación. (2016). Programa Nacional para el aprovechamiento sustentable de la energía 2009-2012. Recuperado de http://dof.gob. $\mathrm{mx} /$ nota_detalle_popup .php?codigo $=5121860$

Eco vehículos. (2016). Manual del usuario del portal de indicadores de eficiencia energética y emisiones vehiculares. Recuperado de: http://www.ecovehiculos.gob.mx/

El informador (2016). Alertan en Jalisco sobre riesgos por contaminación ambiental. Recuperado de http:// www.informador.com.mx/jalisco/2016/656675/6/ alertan-en-jalisco-sobre-riesgos-por-contaminacion-ambiental.htm

González, M. y Asprilla, Y. (2016). La habitabilidad del espacio periurbano en el área metropolitana de Guadalajara: Entropías en la provisión de servicios hidrosanitarios. Tecnogestión: una mirada al ambiente, 13(1), 92-106. doi: http://revistas.udistrital.edu.co/ojs/index.php/tecges/article/ view/12130/12697

González, M. y Jalomo, F. (2017). Expansión periurbana y transformaciones en el espacio geográfico: la recarga hídrica en el Área Metropolitana de Guadalajara. En $11^{\circ}$ Congreso Nacional "Marejadas rurales y luchas por la vida", Bahía de Banderas. Nayarit: México.

González, M. (2016). Infraestructura y desarrollo: las afectaciones del comercio adyacente a línea 3 del tren ligero en Jalisco. Tecnogestión: una 
mirada al ambiente, 13(1), 24-34. doi: http://revistas.udistrital.edu.co/ojs/index.php/tecges/article/ view/12124/12682

González, M. (2017). Movilidad motorizada e infraestructuras de transporte en Culiacán: una situación entrópica. En I.J. Jasso (Coord.). Poder, Cultura y Desarrollo (pp. 60-77). México: Universidad de Guanajuato.

Instituto Nacional de Ecología y Cambio Climático (2014). Evaluación de PM2.5, compuestos orgánicos volátiles y ozono para definir medidas de control en la Zona Metropolitana de Guadalajara: Etapa III. Recuperado de http://www.inecc.gob.mx/descargas/calaire/2014_inf_fin_pm25_guad_e3.pdf

Mendoza, C., Quintero, A., y Alarcón, J. (2015). Algoritmo de gestión para la recarga de vehículos eléctricos. Revista Tecnura, 19, 90-98, doi: http://dx.doi. org/10.14483/udistrital.jour.tecnura.2015.ICE.a11

Nexu (2016). Lo que debes saber sobre cómo funcionan los autos híbridos y eléctricos. Recuperado de http://www.nexu.mx/blog/lo-que-debes-saber-sobre-como-funcionan-los-autos-hibridos-y-electri$\cos /$

ONU-Habitat. (2015). Reporte Nacional de Movilidad Urbana en México 2014-2015. México. Recuperado de http://www.onuhabitat.org/Reporte\%20 Nacional\%20de\%20Movilidad\%20 Urbana $\% 20$ en\%20Mexico\%202014-2015\%20-\%20Final.pdf

Portal Automotriz. (2012). Reporta Melgar de México el parque vehícular en México. Recuperado de http://www.portalautomotriz.com/ noticias/estudios-de-mercado/reportamelgar -de-mexico-el-parque-vehicular-en-mexico

Prigogine, I. (1983). Tan solo una ilusión: una exploración del caos al orden. Barcelona: Tusquest editores.
Ramírez, A. (2016). Mueren seis ciclistas más en 2015 respecto al año anterior. Periódico Milenio. Recuperado de http://www.milenio.com/region/Mueren-ciclistas-respecto-ano-anterior_0_659934037. html

SEMADET. (Secretaria del Medio Ambiente y DesarroIlo Territorial del Estado de Jalisco) (2013). Informe anual de la calidad del aire 2013. Recuperado de http://siga.jalisco.gob.mx/aire/reportes/ReporteAire2013.pdf

SEMADET. (2016). Temporada invernal y la calidad del aire en el AMG 2015-2016. Recuperado de http:// semadet.jalisco.gob.mx/sites/semadet.jalisco.gob. mx/files/temporada _invernal_y_calidad_del_aire_ rp_2015-2016.pdf

Siemiatycki, M. (2006). Message in a Metro. Building Urban Rail Infrastructure and Image in Delhi, India. International Journal of Urban and Regional Research, 2(30), 277-292.

Sipse. (2014). Frena Corte importación de autos chocolate. Recuperado de http://sipse.com/mexico/ importacion-autos-usados-chocolate-suprema-corteamparoexportacio n-116362.html

Valbuena P., García-Ubaque, C., y Granados S., M. (2017). Metodología para el monitoreo estructural y patológico de viviendas afectadas por deslizamientos. Revista Tecnura, 21(52), 79-87, doi: 10.14483/udistrital.jour.tecnura.2017.2.a06

Wiseman, J. (2015). Zero Emissions Plans-Cities and Regions Special Role-Zero Emissions Byron Ambition. Beyond Zero Emissions. doi: http://bze.org.au/me$\mathrm{dia} / \mathrm{ne}$ wswire/zero-emissions-plans-cities-and-regions-special-role-zero-emissions-byron-a151214

\section{(c) $(1) \Theta$}

